\title{
A multivariable prediction model for pegvisomant dosing: monotherapy and in combination with long-acting somatostatin analogues
}

\author{
S E Franck', T I M Korevaar', P Petrossians'2, A F Daly², P Chanson 3,4, Marie-Lise Jaffrain-Rea5, T Brue ${ }^{6,7}$, \\ G K Stalla ${ }^{8}$, D Carvalho ${ }^{9}$, A Colao ${ }^{10}$, V Hána Jr ${ }^{11}$, B Delemer ${ }^{12}$, C Fajardo ${ }^{13}$, A J van der Lely ${ }^{1}$, A Beckers ${ }^{2, *}$ and \\ S J C M M Neggers ${ }^{1, *}$
}

'Department of Internal Medicine, Endocrinology Section, Erasmus University Medical Center, Rotterdam, The Netherlands, ${ }^{2}$ Department of Internal Medicine, Endocrinology Section, Centre Hospitalier Universitaire de Liège, University of Liège, Domaine Universitaire du Sart-Tilman, Liège, Belgium, ${ }^{3}$ Assistance Publique-Hôpitaux de Paris, Hôpitaux Universitaires Paris-Sud, Hôpital de Bicêtre, Service d'Endocrinologie et des Maladies de la Reproduction, Le Kremlin Bicêtre, France, ${ }^{4}$ Inserm 1185, Fac Med Paris Sud, Univ Paris-Sud, Université Paris-Saclay, Le Kremlin-Bicêtre, France, ${ }^{5}$ Department of Biotechnological and Applied Clinical Sciences, University of L'Aquila, L'Aquila and Neuromed, IRCCS, Pozzilli, Italy, ${ }^{6}$ Aix-Marseille Université, CNRS, CRN2M UMR 7286, Marseille, France, ${ }^{7}$ APHM, Hôpital Conception, Service d'Endocrinologie, Diabète et Maladies Métaboliques, Centre de Référence des Maladies Rares d'Origine Hypophysaire, Marseille, France, ${ }^{8} \mathrm{Clinical}$ Neuroendocrinology, Max-Planck-Institute of Psychiatry, Munich, Germany, ${ }^{9}$ Department of Endocrinology, Diabetes and Metabolism Section and Instituto de Investigação e Inovação em Saúde, University of Porto, Centro Hospitalar S. João, Porto, Portugal, ${ }^{10}$ Dipartimento di Medicina Clinica e Chirurgia, Università Federico II di Napoli, Naples, Italy, ${ }^{113 r d ~ D e p a r t m e n t ~ o f ~ I n t e r n a l ~ M e d i c i n e, ~ F i r s t ~ M e d i c a l ~ F a c u l t y, ~ C h a r l e s ~ U n i v e r s i t y, ~ P r a g u e, ~ C z e c h ~ R e p u b l i c, ~}{ }^{12}$ Department of Endocrinology, Diabetes, and Nutrition, University Hospital of Reims, Reims, France, and ${ }^{13}$ Servicio de Endocrinología, Hospital Universitario La Ribera, Valencia, Spain *(A Beckers and S J C M M Neggers contributed equally and share last authorship)

Correspondence should be addressed to S E Franck Email s.franck@erasmusmc.nl

\begin{abstract}
Background: Effective treatment of acromegaly with pegvisomant (PEGV), a growth hormone receptor antagonist, requires an appropriate dose titration. PEGV doses vary widely among individual patients, and various covariates may affect its dosing and pharmacokinetics.

Objective: To identify predictors of the PEGV dose required to normalize insulin-like growth factor I (IGF-I) levels during PEGV monotherapy and in combination with long-acting somatostatin analogues (LA-SSAs).

Design: Two retrospective cohorts (Rotterdam + Liège Acromegaly Survey (LAS), total $n=188$ ) were meta-analyzed as a form of external replication to study the predictors of PEGV dosing in addition to LA-SSA, the LAS ( $n=83$ ) was used to study the predictors of PEGV monotherapy dosing. Multivariable regression models were used to identify predictors of the PEGV dose required to normalize IGF-I levels.

Results: For PEGV dosing in combination with LA-SSA, IGF-I levels, weight, height and age, were associated with the PEGV normalization dosage $(P \leq 0.001, P \leq 0.001, P=0.028$ and $P=0.047$ respectively). Taken together, these characteristics predicted the PEGV normalization dose correctly in $63.3 \%$ of all patients within a range of $\pm 60 \mathrm{mg} / \mathrm{week}(21.3 \%$ within a range of $\pm 20 \mathrm{mg} /$ week). For monotherapy, only weight was associated with the PEGV normalization dose $(P \leq 0.001)$ and predicted this dosage correctly in $77.1 \%$ of all patients within a range of $\pm 60 \mathrm{mg} /$ week $(31.3 \%$ within a range of $\pm 20 \mathrm{mg} / \mathrm{week}$ ). Conclusion: In this study, we show that IGF-I levels, weight, height and age can contribute to define the optimal PEGV dose to normalize IGF-I levels in addition to LA-SSA. For PEGV monotherapy, only the patient's weight was associated with the IGF-I normalization PEGV dosage.

() 2017 European Society of Endocrinology Printed in Great Britain
Published by Bioscientifica Ltd. 


\section{Introduction}

Acromegaly is a rare disease caused by excessive secretion of growth hormone $(\mathrm{GH})$, and a subsequent increase in IGF-I production (1). The disease is almost exclusively caused by a GH-secreting pituitary adenoma (2). Severity and phenotype of the disease varies among acromegaly patients. Uncontrolled acromegaly is associated with an increase in morbidity and mortality (1). The control of IGF-I levels results in mortality rates similar to the general population (3). Although often unsuccessful in macroadenomas, transsphenoidal surgery generally is considered as the first treatment modality $(4,5)$. Additional treatment after surgery is necessary when GH and IGF-I levels remain uncontrolled. Long-acting somatostatin analogues (LA-SSAs), as adjuvant medical treatment or as primary medical treatment, are regularly prescribed. Several studies addressed the response of LA-SSA and show that LA-SSA treatment alone reaches control of the disease in about $40 \%$ of the patients $(6$, 7). A highly effective alternative for patients who are not normalized by LA-SSA monotherapy is the addition of pegvisomant (PEGV) to LA-SSA, or PEGV monotherapy, provided that the appropriate PEGV dose is given $(8,9$, $10,11,12)$. PEGV is a PEGylated recombinant analogue of $\mathrm{GH}$, which competitively blocks the $\mathrm{GH}$ receptor, and thereby reduces the excessive GH actions in the liver and peripheral tissues $(13,14)$. PEGV is slowly absorbed from the subcutaneous depot ( $T_{\max }$ of $33-77 \mathrm{~h}$ and $T_{1 / 2}$ el 74-172h) (15). The mode of PEGV clearance is still not understood. We do not know whether the kidneys and/or the liver metabolize the drug.

The dose of PEGV required to achieve disease control, defined as normalization of IGF-I levels, differs between individual acromegaly patients, both during PEGV monotherapy and in combination with LA-SSA (8, 12). PEGV doses range widely between 20 and $200 \mathrm{mg} /$ weekly during combination treatment with LA-SSA (16). A study by Freda et al. observed that patients using PEGV monotherapy in the ACROSTUDY with persistently elevated IGF-I levels needed a higher mean PEGV dosage (17). Defining the optimal starting dose for PEGV is difficult as the pharmacokinetics remain to be elucidated and data on pre-treatment determinants of the PEGV dosage required for biochemical disease control are sparse. Currently, IGF-I levels are most commonly used during PEGV titration, which is in line with a previous study from our group reporting a positive correlation between baseline IGF-I levels and the PEGV dose required for the normalization of IGF-I during combination treatment of
LA-SSA and PEGV $(8,18)$. Other predictors that have been reported are GH levels, sex, body weight and previous radiotherapy $(19,20)$. Two studies previously reported about a GH receptor polymorphism lacking exon 3, which seemed to have an influence as well during PEGV dosing $(21,22)$. However, more recent studies in larger acromegaly cohorts clearly state that this polymorphism has a clinical effect on neither the PEGV response nor the determination of the required PEGV dose $(23,24,25)$.

Given the importance of swift biochemical control in acromegaly but the lack of studies investigating pre-treatment predictors, we aimed to develop a multivariate regression model for predicting the required PEGV dose to achieve normalization of IGF-I levels in acromegaly patients.

\section{Subjects and methods}

\section{Cohorts description}

Patients $(n=271)$ were included from two retrospective cohorts: (1) the Rotterdam cohort and (2) the Liége Acromegaly Survey (LAS) cohort (26). The Rotterdam cohort contains data from acromegaly patients using LA-SSA in combination with PEGV $(n=112)$ collected in the Pituitary Center Rotterdam between 2004 and 2013, previously published in 2014 (8). The LAS cohort ( $n=3194$ from 14 centers) was created using a software tool that enables hospitals throughout Europe to include acromegaly patients and report patient, biochemical and adenoma characteristics (26). For this study, only patients using PEGV monotherapy $(n=83)$ or PEGV in combination with LA-SSA $(n=76)$ were enrolled from 10 different centers. The inclusion period was between 2010 and 2015.

\section{Rotterdam cohort}

Clinical and biochemical data were collected from acromegaly patients with elevated IGF-I levels $(>1.2 \times$ upper limit of normal (ULN)), after at least 6 months of the highest dose of LA-SSAs (octreotide LAR $30 \mathrm{mg}$ or lanreotide Autogel $120 \mathrm{mg}$ every 28 days). In this group, 27 acromegaly patients started with $25 \mathrm{mg}$ PEGV weekly as co-treatment, whereas another 18 started with $40 \mathrm{mg}$ PEGV weekly and the last 67 patients started with a variable PEGV dose, guided by their baseline IGF-I levels. This variable PEGV starting dose was based on one of our previous reports (18)). The formula to calculate the PEGV dose is $4+$ (IGF-I $Z$-score during treatment with high dose 
LA-SSA *16) and was deducted from a method described previously (18). This formula can only be used when IGF-I levels are elevated after a period of at least 6 months of LA-SSA treatment. Intervals of dose adaptations were 6-8 weeks until a controlled IGF-I level was achieved on two consecutive occasions. The subjects then visited our outpatient clinic every 16 weeks. When the once-weekly PEGV dose exceeded $80 \mathrm{mg}$ per injection, patients divided the dosage to two weekly injections. With weekly doses over $200 \mathrm{mg}$, subjects changed administration intervals into daily injections or 5 injections per week. At each visit to our outpatient clinic, standard measurements were performed including assessments of IGF-I levels. Permission from the Institutional Review Board of the Erasmus Medical Center Rotterdam was obtained, and all patients gave their written informed consent.

\section{LAS cohort}

Acromegaly patients from the LAS database treated with PEGV were selected and divided in two groups; PEGV in combination with LA-SSA and PEGV monotherapy. From the LAS database, we were able to select 141 potential patients using the combination treatment. We excluded 65 patients, because of two reasons: (1) no IGF-I normalization during LA-SSA+PEGV treatment was achieved $(n=16)$ and (2) follow-up data during LA-SSA/PEGV treatment were missing $(n=49)$. The remaining patients $(n=76)$ were selected for this study. The same exclusion criteria applied for the PEGV monotherapy patients. We were able to select 122 potential patients using PEGV monotherapy. We excluded 39 patients (no IGF-I normalization during PEGV monotherapy was achieved $(n=6)$ and follow-up data during PEGV treatment were missing $(n=33))$. The remaining patients $(n=83)$ were selected for this study. The medical ethics committee from the Liège University hospital approved the protocol and was covering the other European centers.

\section{Hormone assays}

In the Rotterdam cohort, the GH and IGF-I level measurements were assessed with the IMMULITE 2000 assay (DPC Biermann GmbH/Siemens, Fernwald, Germany), a solid-phase, enzyme-labeled chemiluminescent immunometric assay, with an intraassay variability of $6 \%$, and an inter-assay variability of $5-6 \%$ for GH and with an intra-assay variability of $2-5 \%$, and an inter-assay variability of $3-7 \%$ for IGF-I. The IGF-I age- and sex-adjusted reference ranges were used from an article by Elmlinger et al. (27). In the LAS cohort, containing acromegaly patients from several European hospitals, the GH and IGF-I level measurements were assessed locally and consequently performed with different assays. Therefore, the IGF-I levels were chosen to be expressed as the upper limit of normal (ULN) of the reference ranges used in the local hospitals. In this study, GH levels were measured as a single random sample and expressed as absolute values.

\section{Candidate predictors}

Variables that were considered as possible predictors for PEGV normalization dosage were selected based on the literature $(8,18,19,20)$, biological plausibility and availability of robust data ascertainment in both cohorts and included: age at diagnosis, sex, weight, height, tumor size (micro vs macroadenoma at diagnosis), presence of diabetes mellitus, IGF-I levels (expressed as ULN), random GH levels and previous treatment modalities (transsphenoidal surgery, radiotherapy and the duration of LA-SSA monotherapy before the addition of PEGV). Weight, IGF-I levels (expressed as ULN) and random GH levels were collected between 6 months before and at the time of PEGV addition. Other data were collected at baseline (as indicated), were fixed data in the patient's record or were established during disease process.

\section{Outcome}

The outcome used in this study was the PEGV dose $(\mathrm{mg} /$ week) needed for the normalization of IGF-I levels either during the addition to LA-SSA (highest tolerable dose) or as PEGV monotherapy.

\section{Statistical analysis}

Data are expressed as median (interquartile range). Differences between two subgroups were analyzed using an unpaired $t$-test or the Mann-Whitney $U$ test (in case of non-parametric data). Nominal variables were analyzed using Fisher's exact test. For subjects in which PEGV was added to LA-SSA therapy, the distribution of the PEGV dose required for normalization of IGF-I levels was not comparable between the two cohorts; therefore, we metaanalyzed the data as a form of external replication. For all regression models, log-transformation of the outcome variable (required PEGV dose) was performed to normalize 
residuals, and non-linearity was assessed utilizing restricted cubic splines with 3-4 knots. We used univariable linear regression models to assess the association between each candidate predictor and the required PEGV dose. The decision for linear regression models instead of multiple models for the identification of predictors was based on Akaike information criteria and log-likelihood tests comparing multilevel models with random intercepts and/or slope per cohort vs standard linear regression correcting for cohort. To allow for optimal generalizability of effect estimates that predict the required PEGV dose, we performed multivariable multilevel modeling with a random intercept per cohort for the final model. We selected useful predictors using backward selection based on the change in regression coefficients and residual explained variability of the model, with a $P$ value $<0.20$ as to keep predictors liberally in the model. Other $P$ values are considered statistically significant when lower than 0.05 (two-tailed). For subjects switching from LA-SSA to PEGV monotherapy, we used univariable linear regression models to assess the association between each potential predictor and the required PEGV dose. We subsequently calculated the predicted normalization dosage for each subject using the outcomes of the final (multivariable) regression models. In addition, we also calculated more conservative and more progressive models to cope with potential under or overtreatment by adding or subtracting the equivalent of $40 \mathrm{mg} /$ week from the outcome of the regression formula. To cope with (differentially) missing values of the candidate predictors, missing data on candidate predictors were multiple imputed (five times). The imputation model included all candidate predictor variables, the outcome variable and several relevant variables descriptive for the study subjects. There was no difference between the original or any of the imputed datasets. All analyses were performed in each of the completed datasets, and final results were pooled. All statistical analyses were performed using Statistical Package of Social Sciences, version 20.0 for Windows (SPSS) or using R statistical software, version 3.2.43 (packages rms, MASS and $\operatorname{lm} 4$ ).

Table 1 Descriptive characteristics of the combination treatment and PEGV monotherapy cohorts. Descriptive characteristics of the three cohorts: Rotterdam cohort using LA-SSA + PEGV, LAS cohort using LA-SSA + PEGV and the LAS cohort using PEGV monotherapy. Missing data were imput into the original datasets by multiple imputation. Continuous variables are expressed in median (interquartile range) and categorical variables in percentages.

\begin{tabular}{|c|c|c|c|c|c|c|}
\hline & \multicolumn{3}{|c|}{ Combination treatment (LA-SSA+PEGV) } & \multirow{2}{*}{$\begin{array}{c}P \\
\text { value }^{a}\end{array}$} & \multirow{2}{*}{$\frac{\text { PEGV monotherapy }}{\text { LAS }}$} & \multirow{2}{*}{$\begin{array}{c}P \\
\text { value }\end{array}$} \\
\hline & Total cohort & Rotterdam & LAS & & & \\
\hline No. of patients & 188 & 112 & 76 & & 83 & \\
\hline \multicolumn{7}{|l|}{ Patient characteristics } \\
\hline Age at diagnosis & $42.0(33.0-53.0)$ & $45.5(36.0-56.0)$ & $39.0(29.5-47.0)$ & 0.000 & $41.0(29.0-51.0)$ & 0.001 \\
\hline Sex $($ Male $(\%))$ & 58.0 & 58.0 & 57.9 & 1.000 & 53.0 & 0.000 \\
\hline $\begin{array}{l}\text { Weight before addition of } \\
\text { PEGV }(\mathrm{kg})\end{array}$ & $90.0(77.0-104.0)$ & $91.5(79.0-104.0)$ & $89.0(74.5-105.0)$ & 0.107 & $83.0(71.0-93.0)$ & 0.000 \\
\hline $\begin{array}{l}\text { Height before addition of } \\
\text { PEGV }(\mathrm{cm})\end{array}$ & $175.0(168.0-182.0)$ & $178.0(170.0-184.0)$ & $170.0(166.0-180.0)$ & 0.000 & $170.0(163.0-180.0)$ & 0.000 \\
\hline Tumor size (Macro (\%)) & 85.1 & 81.3 & 90.8 & 0.000 & 83.9 & 0.276 \\
\hline Diabetes Mellitus (\%) & 39.4 & 36.6 & 43.4 & 0.025 & 34.9 & 0.050 \\
\hline $\begin{array}{l}\text { IGF-I xULN before addition } \\
\text { of PEGV }\end{array}$ & $2.0(1.5-2.7)$ & $1.9(1.5-2.6)$ & $2.1(1.6-2.8)$ & 0.000 & $2.1(1.5-3.2)$ & 0.001 \\
\hline $\begin{array}{l}\text { GH before addition of PEGV } \\
(\mu \mathrm{g} / \mathrm{L})\end{array}$ & $7.9(3.1-17.8)$ & $8.4(3.2-17.5)$ & $7.5(2.2-18.6)$ & 0.617 & $5.9(2.0-11.0)$ & 0.000 \\
\hline \multicolumn{7}{|l|}{ Previous treatment } \\
\hline Surgery (total (\%)) & 51.0 & 28.6 & 84.2 & 0.000 & 81.9 & 0.000 \\
\hline Once debulked (\%) & 48.6 & 28.6 & 78.1 & & 71.1 & \\
\hline Twice debulked (\%) & 2.4 & N/A & 6.1 & & 8.4 & \\
\hline >Twice debulked (\%) & N/A & N/A & N/A & & 2.4 & \\
\hline $\mathrm{RTx}(\%)$ & 16.0 & 10.7 & 23.7 & 0.000 & 40.2 & 0.000 \\
\hline $\begin{array}{l}\text { Duration of LA-SSA before } \\
\text { addition of PEGV (months) }\end{array}$ & $16.0(8.3-39.0)$ & $12.0(7.2-26.8)$ & $25.0(11.5-62.0)$ & 0.000 & $34.4(13.4-86.4)$ & 0.000 \\
\hline \multicolumn{7}{|l|}{ Outcome } \\
\hline $\begin{array}{l}\text { Required PEGV dose (mg } \\
\text { weekly) }\end{array}$ & $105.0(65.0-200)$ & $80.0(60.0-120.0)$ & $210.0(105.0-280.0)$ & 0.000 & $105.0(105-140)$ & 0.000 \\
\hline
\end{tabular}

www.eje-online.org 


\section{Results}

\section{Cohort characteristics}

Patient characteristics and previous treatment modalities of the two combination treatment cohorts and the PEGV monotherapy cohort are depicted in Table 1 . Acromegaly patients treated with the combination treatment included in the LAS database are younger (39.0 vs 45.5 years), more likely to be diagnosed with a macroadenoma $(90.8 \%$ vs $81.3 \%)$ and suffered from diabetes mellitus more frequently $(43.4 \%$ vs $36.6 \%)$. Patients from the Rotterdam cohort are taller $(178$ vs $170 \mathrm{~cm})$. Patients who were included in the LAS database needed higher PEGV doses to achieve normalized IGF-I levels both during combination treatment with LA-SSA and during PEGV monotherapy and had a higher IGF-I level ( $x$ ULN) before the addition of PEGV. Other descriptive data and measurements such as weight, height and biochemical data are depicted in Table 1, as well as comparisons between the combination treatment group and the PEGV monotherapy group. No significant differences were observed in the combination treatment cohort between excluded (all originated from the LAS database) and included patients, except for the percentage of performed surgeries, radiotherapy and height, the excluded patients were smaller in stature. No significant differences were observed in the PEGV monotherapy cohort between excluded and included patients.

\section{Predictors of PEGV dosing required for disease control in combination treatment with LA-SSA}

All univariate analyses of the candidate predictors are depicted in Fig. 1. A positive linear association was observed between IGF-I $(\times \mathrm{ULN})$ and the PEGV dosage required for disease control. There was a positive nonlinear association of weight with PEGV normalization dosage, suggesting an effect threshold from approximately $100 \mathrm{~kg}$ (Fig. 1); results were similar after adjustment for age and height (data not shown). There was a negative linear association of age with PEGV normalization dosage and a positive linear association of height with PEGV normalization dosage. In multivariable analyses, the association of age and height were no longer statistically significant after adjustment for weight; yet, age did meet the pre-specified criteria of being added in the final model. Other potential predictors were not associated with the PEGV normalization dosage (Fig. 1).

Figure 2 depicts the performance of the standard prediction model ( $X$-axis) as compared to the true PEGV normalization dosage ( $Y$-axis) and the difference between the predicted and true normalization PEGV dose for each individual (colored dots are corresponding to the table colors; Fig. 2). The standard prediction formula for PEGV normalization dosage based on multivariable models $\left(\operatorname{EXP}^{\wedge}\left(5.5994+\mathrm{IGF}-1 \quad \mathrm{ULN}^{*} 0.2585+\right.\right.$ weight $*-0.0365+$ weight ${ }^{2 *} 0.00025+$ age $\left.{ }^{*}-0.0045\right)$ ) (Table 2) predicted the final PEGV normalization dose correctly in $63.3 \%$ of all
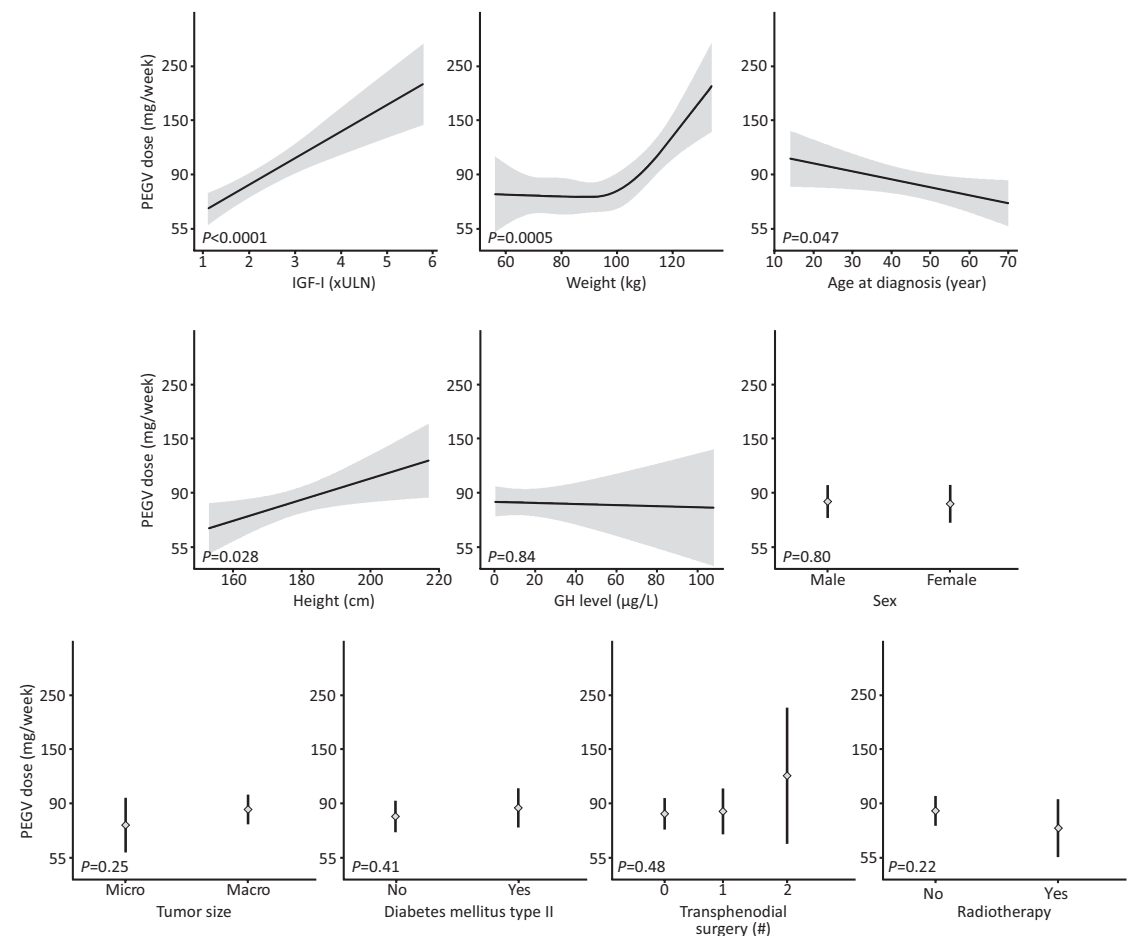

\section{Figure 1}

Identification of potential predictors during combination treatment. Univariate analyses of multiple determinants potential for the prediction of the PEGV dose needed to achieve normalization of IGF-I levels during combination treatment. IGF-I $\times$ ULN, age at diagnosis, weight and height were significantly associated with PEGV dosing during PEGV treatment in combination with LA-SSA. GH, growth hormone; IGF-I, insulin-like growth factor I; macro, macroadenoma; micro, microadenoma; PEGV, pegvisomant; ULN, upper limit of normal. 

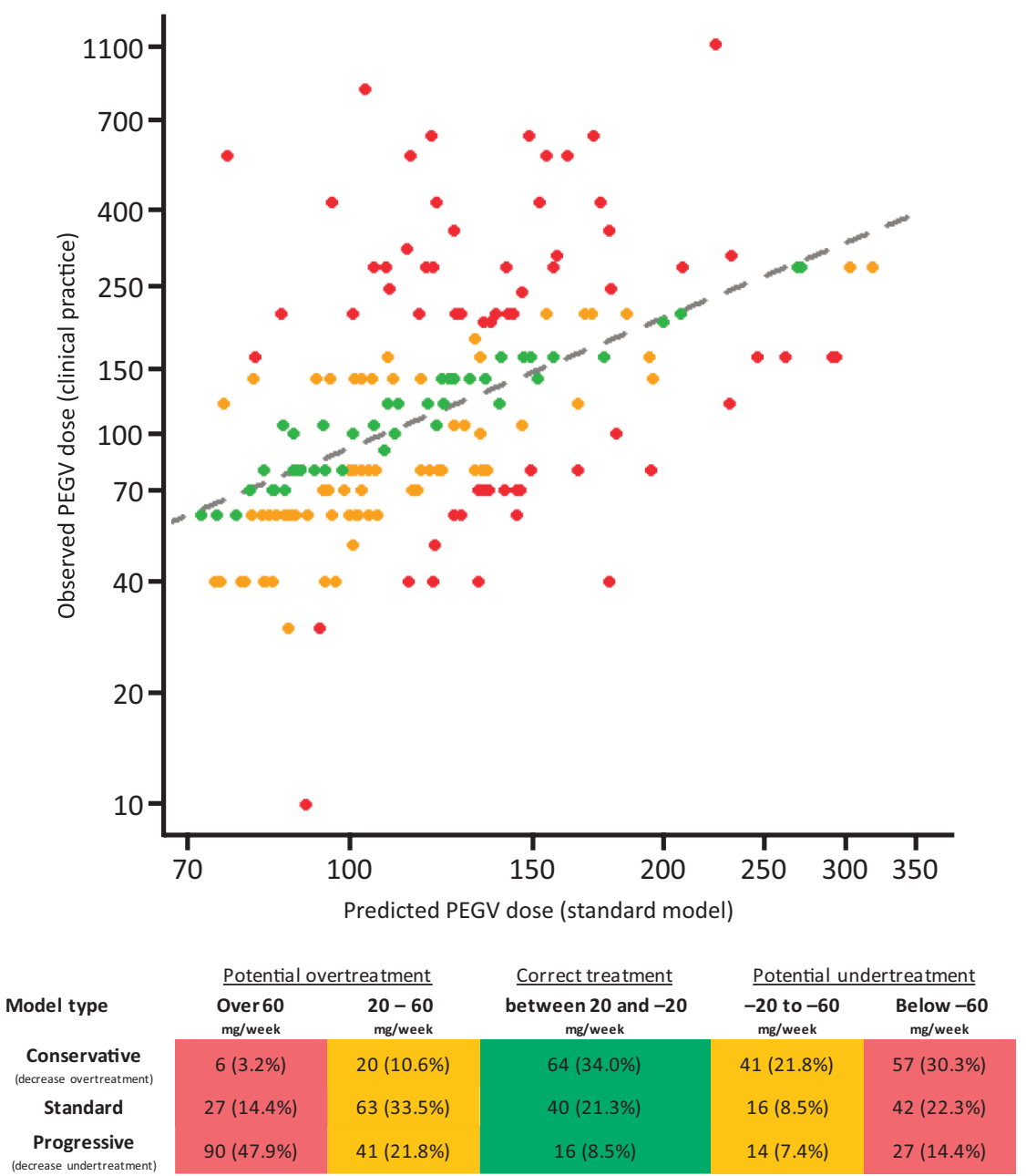

\section{Figure 2}

Association of combined predictive values with the PEGV dose needed for IGF-I normalization. This figure shows the association of the combined predictive values ( $X$-axis, the model) with the PEGV dose needed for IGF-I normalization as obtained in clinical practice ( $Y$-axis). The regression line is represented by the dashed line (gray). The individual data points are colored according to the distance from the regression line (red: distance $=60 \mathrm{mg} / \mathrm{week}$, orange $20-60 \mathrm{mg} /$ week, green $<20 \mathrm{mg} /$ week). Data points in the figure depict the standard model. The conservative and progressive model were defined as the normal model minus or plus $40 \mathrm{mg} /$ week respectively. The table below depicts the $n(\%)$ of the different model groups and also display the potential shift between the models. patients within a range of $\pm 60 \mathrm{mg} /$ week and in $21.3 \%$ of all patients within a range of $\pm 20 \mathrm{mg} /$ week (Fig. 2). In addition, a more conservative model (standard prediction model minus $40 \mathrm{mg} /$ week) correctly predicted the PEGV normalization dosage in $66.4 \%$ of all patients within a range of $\pm 60 \mathrm{mg} /$ week and in $34.0 \%$ of all patients within a range of $\pm 20 \mathrm{mg} /$ week (Fig. 2). For a more progressive

Table 2 Multivariable analysis of final model to predict optimal PEGV dosing.

\begin{tabular}{|c|c|c|c|}
\hline Variable & Estimate & SE & $P$ value \\
\hline Intercept & 5.5994 & 0.9382 & $<0.0001$ \\
\hline IGF-I (xULN)* & 0.2585 & 0.0459 & $<0.0001$ \\
\hline Weight (kg)* & -0.0365 & 0.0192 & 0.0830 \\
\hline Weight $^{2}(\mathrm{~kg})$ * & 0.0002 & 0.0001 & 0.0038 \\
\hline Age at diagnosis (years) & -0.0045 & 0.0033 & 0.1700 \\
\hline
\end{tabular}

As the outcome is not normally distributed, the model should be calculated as: $e^{(\text {final model) }}$

*Before the addition of PEGV to LA-SSA.

IGF-I, insulin-like growth hormone I; PEGV, pegvisomant; SE, standard error; ULN, upper limit of normal. model (standard model plus $40 \mathrm{mg} /$ weekly), these numbers were $37.7 \%$ and $8.5 \%$ respectively (Fig. 2 ).

\section{Predictors of PEGV dosing required for disease control during PEGV monotherapy}

A positive linear association was observed between weight and the PEGV dosage required for disease control $(P \leq 0.001$; Figs 3 and 4$)$. None of the other potential predictors were associated with the PEGV normalization dosage (Fig. 3). Figure 4 depicts the performance of weight ( $X$-axis) as a predictor for PEGV normalization dosage as compared to the true normalization dosage ( $Y$-axis) and the difference between the predicted and true normalization dosage for each individual (colored dots are corresponding to the table colors; Fig. 4). The standard prediction formula for PEGV normalization dosage based on weight $\left(\mathrm{EXP}^{\wedge}\left(4.092+\right.\right.$ weight $\left.\left.{ }^{*} 0.00868\right)\right)$ predicted the final PEGV normalization dose correctly in $77.1 \%$ of all patients within a range of $\pm 60 \mathrm{mg} /$ week and in $31.3 \%$ of 

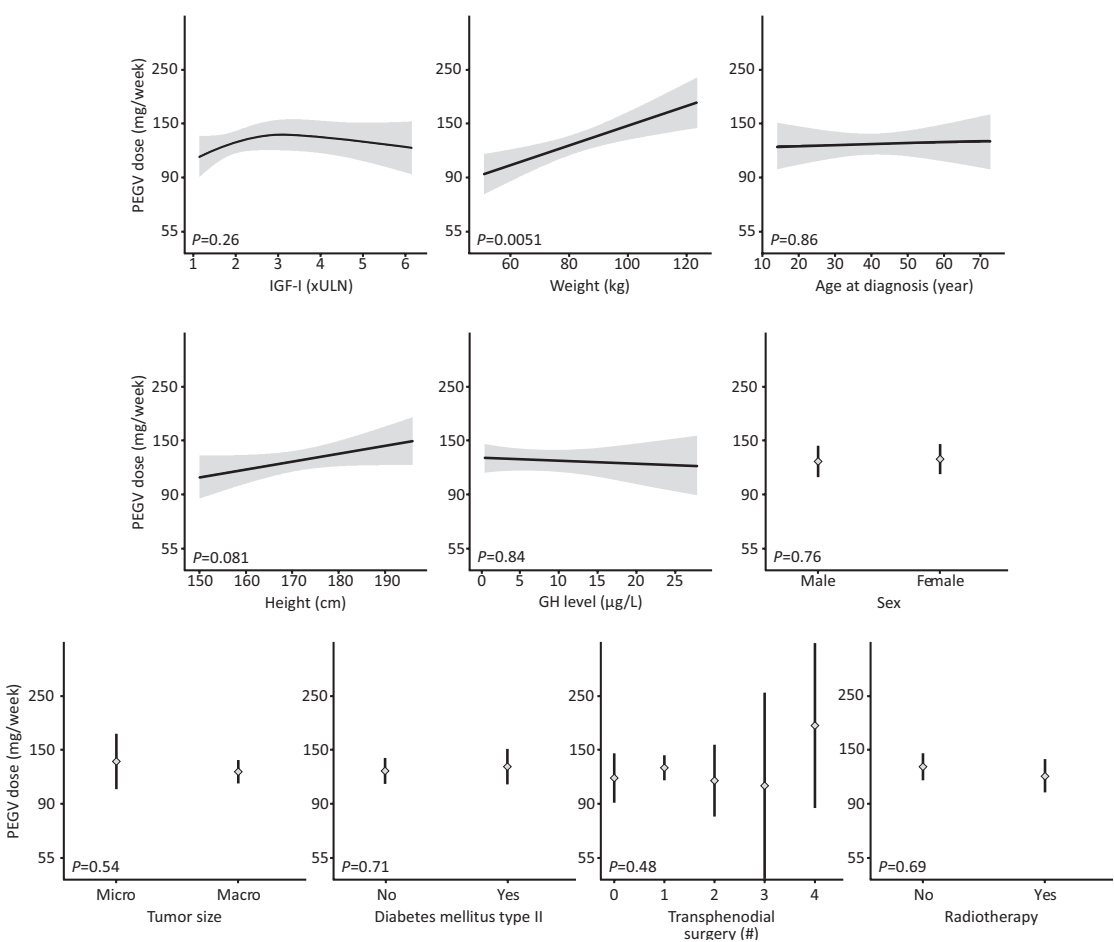

\section{Figure 3}

Identification of potential predictors during PEGV monotherapy. Univariate analyses of multiple determinants potential for the prediction of the PEGV dose needed to achieve normalization of IGF-I levels during PEGV monotherapy. Only weight was significantly associated with PEGV dosing during PEGV monotherapy. GH, growth hormone; IGF-I, insulin-like growth factor I; macro, macroadenoma; micro, microadenoma; PEGV, pegvisomant; ULN, upper limit of normal. all patients within a range of $\pm 20 \mathrm{mg} /$ week (Fig. 4). In addition, a more conservative model correctly predicted the PEGV normalization dosage in $67.4 \%$ of all patients within a range $\pm 60 \mathrm{mg} /$ week and in $32.5 \%$ of all patients within a range of $\pm 20 \mathrm{mg}$ /week. For a more progressive model, these numbers were $56.6 \%$ and $14.5 \%$ respectively.

\section{Discussion}

The PEGV dose required for normalization of IGF-I levels in acromegaly is highly variable and a wide inter-individual variation in PEGV serum levels is observed despite identical PEGV dosage $(28,29)$. Previous studies suggest that this variability depends on disease activity and individual response to the drug $(8,16)$. Therefore, PEGV titration is a process that requires a tailored approach for each individual. This is the first study that focuses on identifying predictors for PEGV dosing and developing a multivariable model to predict the required PEGV dose to achieve normalization of IGF-I levels in acromegaly patients. The main findings of this study are (1) IGF-I, weight, height and age at diagnosis are associated with the PEGV dose required for normalization of IGF-I levels in patients treated with LA-SSA combined with PEGV and (2) that weight is associated with the PEGV dose required for normalization of IGF-I levels in patients treated with PEGV monotherapy.
To the best of our knowledge, only one previous study has investigated determinants of the PEGV dose needed for IGF-I normalization. Parkinson et al. observed that GH and IGF-I levels, sex, weight and previous radiotherapy were associated with the PEGV dose required for disease control in patients treated with PEGV monotherapy $(n=118)$ (20). In our study, IGF-I $\times$ ULN was the best predictor for PEGV dosing; yet, GH levels were not associated with the required PEGV dose. The most likely explanation for this difference is the variability of the GH assays. The study by Parkinson et al. used a single assay for the measurement of all GH levels, whereas GH levels in our study were measured in several local hospitals and thereby consequently measured by different GH assays. This can lead to measurement errors and a bias. Moreover, single GH has a limited clinical usefulness as it has a short half-life and is pulsatile excreted into the bloodstream. Therefore, random single measurements of GH are less suitable as a biochemical marker for acromegaly in clinical practice. These aspects are less prominent for IGF-I measurements, as they are expressed as the upper limit of normal and are less sensitive to daily variations as compared to GH. Despite the limitations of GH-measurement, we chose to include and analyze these GH levels because of its biological plausibility as a candidate predictor and the intension that our prediction model is going to be used in multiple hospitals and consequently GH measurements will be performed with several different assays. 

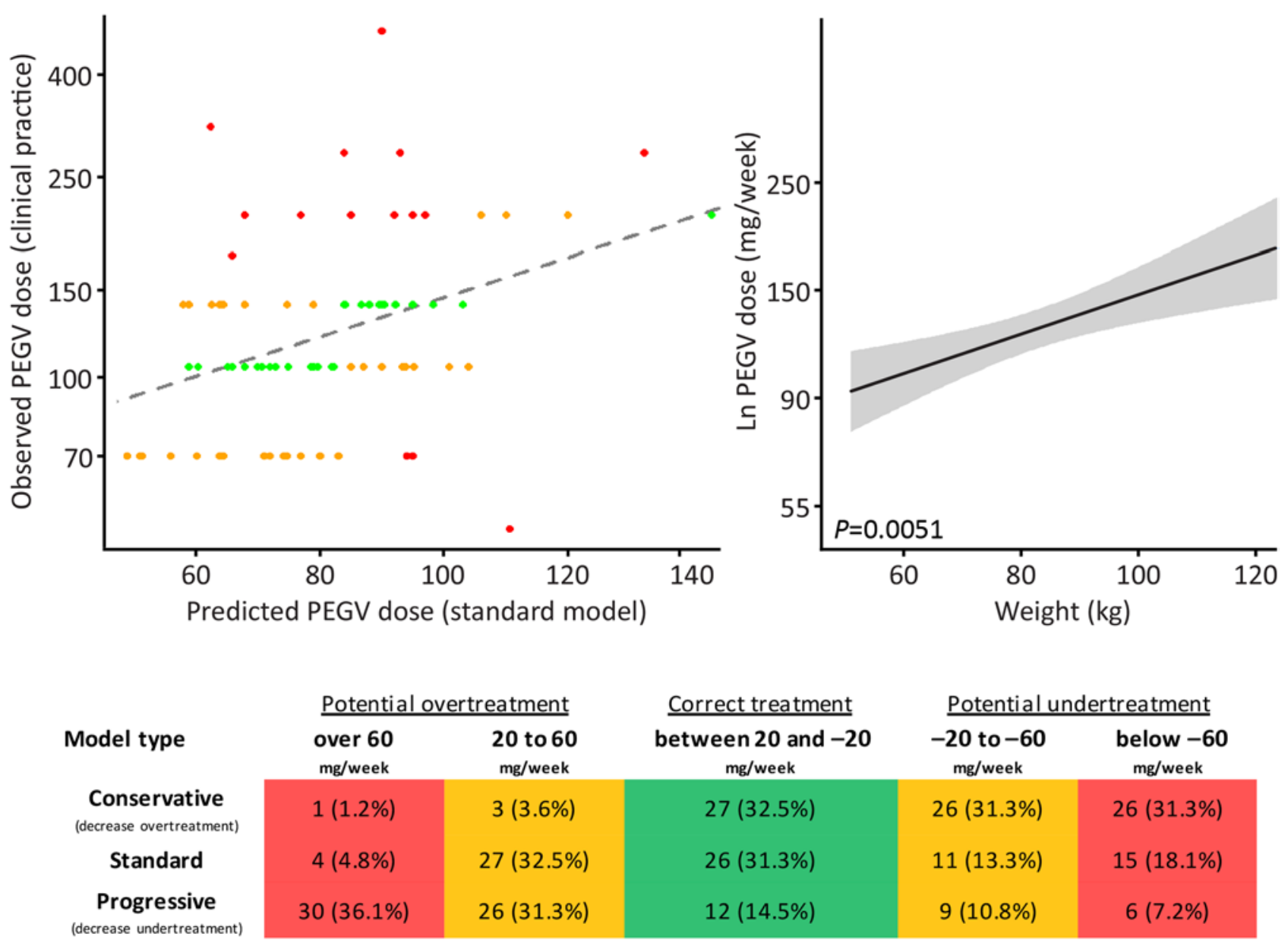

Potential overtreatment over $60 \quad 20$ to 60

$\mathrm{mg} /$ week

$\mathrm{mg} /$ week

$\mathrm{mg} /$ week

0 to -60

$1(1.2 \%) \quad 3(3.6 \%)$

27 (32.5\%)

$6(31.3 \%)$

$\mathrm{mg} /$ week

$4(4.8 \%)$

27 (32.5\%)

$26(31.3 \%)$

$11(13.3 \%)$

$26(31.3 \%)$

$30(36.1 \%)$

$26(31.3 \%)$

$12(14.5 \%)$

$9(10.8 \%)$

$15(18.1 \%)$

$6(7.2 \%)$

\section{Figure 4}

Association of weight with the PEGV dose needed for IGF-I normalization. This figure shows the association of the patient's weight (X-axis) with the PEGV dose needed for disease control as obtained in clinical practice (Y-axis). The regression line is represented by the dashed line (gray). The individual data points are colored according to the distance from the regression line (red: distance $=60 \mathrm{mg} /$ week, orange $20-60 \mathrm{mg} /$ week and green $<20 \mathrm{mg} /$ week). Data points in the figure depict the standard model. The conservative and progressive models were defined as the normal model minus or plus $40 \mathrm{mg} / \mathrm{week}$ respectively. The table below depicts the $\mathrm{n}(\%)$ of the different model groups and also displays the potential shift between the models.

The best predictor during combination treatment, besides IGF-I, is the patients weight before the start of PEGV. Patients with a higher bodyweight, require a higher PEGV dosage, which is a logical and expected phenomenon. However, in our study, a positive nonlinear association was observed, suggesting a threshold effect from approximately $100 \mathrm{~kg}$ body weight, which remained similar after correction for sex, age and IGF-I levels. A possible explanation for this effect threshold could be that these patients have different disease activity and therefore have a different body composition, possibly more fat mass. Former studies already reported an association between weight and PEGV dose titration
$(19,20,30)$. Future studies should investigate whether a clinical assessment of body composition (ratios of lean vs fat mass percentages) may improve the prediction of the PEGV dose required for biochemical normalization.

Female gender is reported to have a better PEGV response with similar PEGV doses during PEGV monotherapy; however, this gender difference was not statically significant anymore when PEGV doses were expressed per kg body weight (19). Another study did observe that women needed a higher average PEGV dose of $0.04 \mathrm{mg} / \mathrm{kg} /$ day during PEGV monotherapy (20). It has been speculated that sex differences in PEGV pharmacokinetics may influence absorption, distribution 
and/or clearance of the drug as well as the modulation of GH sensitivity by estrogens and fat $(31,32,33)$. However, regardless of weight differences, we could not confirm a sex difference in relation to the PEGV normalization dose during our study both in patients treated with PEGV monotherapy and in combination with LA-SSA.

Opposite to patients treated with the combination therapy, we found that IGF-I was not a predictor of PEGV dosing during PEGV monotherapy, despite its biological plausibility. This may be explained by differences in the disease severity of patients in the combination vs monotherapy groups, given that the LAS combination cohort requires a median PEGV dose of $210 \mathrm{mg}$ /week on top of the maximum LA-SSA dosage, whereas the LAS cohort treated with PEGV monotherapy required a median dose of $105 \mathrm{mg} /$ week. According to the literature, to achieve efficacy rates of more than 90\% during PEGV monotherapy, the average expected weekly dose is above $120-130 \mathrm{mg}(12,34)$. Studies about the combination treatment reported PEGV doses that range between 60 and $140 \mathrm{mg}$ weekly in addition to LA-SSA (normalization rates range between $67 \%$ and $97 \%)(8,10,35)$. These data show that the LAS monotherapy group contains less severe acromegaly patients, whereas the LAS combination treatment group contains more severe acromegaly patients relative to data from the literature, presumed that the PEGV dose represents disease severity. On the other hand, LA-SSA has a direct and an indirect effect, which results in GH-independent decrease of IGF-I secretion $(36,37)$. A Danish group observed that PEGV serum levels increase by $20 \%$ when combined with LA-SSA (38). Besides dosing difference, it may be expected that the use of two drug modalities is naturally more given to patients with more disease severity. Additionally, IGF-I $(\times \mathrm{ULN})$ levels before the addition of PEGV in both LAS cohorts treated with monotherapy and combination treatment are higher. On the other hand, the differences between the various IGFI-assays that were used in the different cohorts should be taken into account.

The PEGV doses of the LAS cohort required for IGF-I normalization were strikingly high compared to those of the Rotterdam cohort. The distribution of normalization PEGV dosage was right skewed as opposed to the normally distributed Rotterdam cohort. This most likely reflects the fact that the LAS cohort represents the more severe cases in Europe, whereas the experience with PEGV in Rotterdam has led to a relatively low threshold for prescribing PEGV in addition to LA-SSA. This may not be directly linked to a difference in IGF-I levels before the addition of PEGV in our study; however, LAS patients are younger and are having more diabetes mellitus, which are the characteristics of more severe acromegaly. Another possible explanation could be the interest of the research group in Liège for genetic disorders causing acromegaly, taking into account that the possible prevalence of a mutation in the aryl hydrocarbon receptor-interacting protein (AIP) gene, $\mathrm{X}$-linked acrogigantism (X-LAG) and/or familial isolated pituitary adenoma (FIPA) patients could be higher in this cohort. Despite these differences, we found that a metaanalysis of both cohorts (as a form of external replication) performed well and also the separate analyses per cohort showed the same effect directions. By combining both cohorts, the results of this study are widely generalizable as this approach has led to a study population that reflects a wide range of acromegaly patient that is eligible to start PEGV treatment.

This study was potentially limited by the retrospective design, which consequently led to missing data. To cope with both differentially and randomly missing data, we used multiple imputation. This study was also limited by the relative small sample size. However, this is expected given the low prevalence of acromegaly as well as the fact that only a subset of acromegaly patients is treated with LA-SSA in combination with PEGV. The Rotterdam cohort harbored exclusively patients who were normalized by LA-SSA in combination with PEGV, as PEGV doses were uptitrated until normalization of IGF-I levels was achieved. The exclusion of patients from the LAS cohort not normalized by LA-SSA and PEGV $(n=16,8.5 \%)$ or PEGV alone $(n=6,7.2 \%)$ has remained limited. To overcome these limitations and to replicate our results, prospective studies utilizing a multicenter set-up are required.

This model is designed for patients who are about to start PEGV treatment after failure of LA-SSA monotherapy. Furthermore, this study is not designed to predict PEGV overdosing as PEGV doses were increased until IGF-I levels were normalized. However, this prediction model should be considered as a useful clinical tool during PEGV dose titration, which can be time consuming over multiple outpatient clinic visits, especially when a high PEGV dose is needed to control the disease.

\section{Conclusion}

This is the first study that focuses on identifying predictors for the PEGV dose required for disease control in acromegaly and the development of a multivariate prediction model for the required PEGV dose. The model is designed for 
patients who are about to start PEGV after failure of LA-SSA monotherapy and could be used as a clinical guidance tool during the start of PEGV dose titration. In this study, the PEGV dose needed for normalization of IGF-I levels in addition to LA-SSA is associated with IGF-I levels, weight and age in a multivariate prediction model and predicted the final PEGV normalization dose correctly in $63.3 \%$ of all patients within a range of $\pm 60 \mathrm{mg} /$ week ( $21.3 \%$ within a range of $\pm 20 \mathrm{mg}$ /week). The required PEGV dose during monotherapy was associated with the patient's weight and predicted the final PEGV normalization dose correctly in $77.1 \%$ of all patients within a range of $\pm 60 \mathrm{mg}$ /week (31.3\% within a range of $\pm 20 \mathrm{mg} /$ week). For an acromegaly patient of age 60 years, weight of $80 \mathrm{~kg}$, height of $1.75 \mathrm{~m}$ and a IGF-I level of $1.6 \times$ the ULN using the maximum dose of LA-SSA, the standard model will calculate $83.3 \mathrm{mg}$ PEGV weekly. In this case, we will recommend to start with $80 \mathrm{mg}$ weekly and titrate up or down guided by the IGF-I level (target $1.0 \times$ the ULN).

\section{Declaration of interest}

A J van der Lely and S J C M M Neggers have received financial support for investigator-initiated research, unrestricted grants and speaker's fees from Novartis, Pfizer and Ipsen. A J van der Lely also received consultancy fees from Novartis and Pfizer. P Petrossians have received consultancy fees from Novartis, Ipsen and Pfizer. P Chanson has received unrestricted research and educational grants from Ipsen, Novartis and Pfizer as Head of the Department of Endocrinology and Reproductive Diseases, Hôpitaux Universitaires Paris-Sud, he has served as investigator (principal or coordinator) for clinical trials funded by Novartis, Pfizer, Ipsen, Italfarmaco and Antisense, he is member of Advisory Boards from Ipsen and Novartis and he gave lectures for Ipsen, Novartis and Pfizer. All the fees and honoraria are paid to his institution. $M L$ Jaffrain-Réa received a consultancy fee from Novartis. T Brue received financial support research grants, consultant or speaker fees from Ipsen, Novartis, Pfizer, Sandoz and Strongbridge. A Colao is a member of the Italian scientific board of Pfizer. C Fajardo has received honoraria from Pfizer as speaker fees. A Beckers has received educational grant funding from Pfizer and Ipsen and Advisory Board Membership for Ipsen. S E Franck, T I M Korevaar, A F Daly, G K Stalla, D Carvalho, V Hána Jr. and B Delemer have nothing to disclose.

\section{Funding}

This work did not receive any specific grant from any funding agency in the public, commercial or not-for-profit sector. The Liège Acromegaly Survey (LAS) was developed with an unrestricted educational grant from Ipsen to A Beckers.

\section{Acknowledgements}

The authors would like to thank all patients for their participation.

\section{References}

1 Melmed S. Acromegaly pathogenesis and treatment. Journal of Clinical Investigation 2009 119 3189-3202. (doi:10.1172/JCI39375)
2 Melmed S. Medical progress: acromegaly. New England Journal of Medicine 2006355 2558-2573. (doi:10.1056/NEJMra062453)

3 Holdaway IM, Bolland MJ \& Gamble GD. A meta-analysis of the effect of lowering serum levels of GH and IGF-I on mortality in acromegaly. European Journal of Endocrinology 2008159 89-95. (doi:10.1530/EJE08-0267)

4 Bates PR, Carson MN, Trainer PJ, Wass JA \& UK National Acromegaly Register Study Group (UKAR-2). Wide variation in surgical outcomes for acromegaly in the UK. Clinical Endocrinology 200868 136-142. (doi:10.1111/j.1365-2265.2007.03012.x)

5 Holdaway IM. Treatment of acromegaly. Hormone Research 200462 (Supplement 3) 79-92. (doi:10.1159/000080505)

6 Annamalai AK, Webb A, Kandasamy N, Elkhawad M, Moir S, Khan F, Maki-Petaja K, Gayton EL, Strey CH, O’Toole S et al. A comprehensive study of clinical, biochemical, radiological, vascular, cardiac, and sleep parameters in an unselected cohort of patients with acromegaly undergoing presurgical somatostatin receptor ligand therapy. Journal of Clinical Endocrinology and Metabolism 201398 1040-1050. (doi:10.1210/jc.2012-3072)

7 Caron PJ, Bevan JS, Petersenn S, Flanagan D, Tabarin A, Prevost G, Maisonobe P, Clermont A \& Investigators P. Tumor shrinkage with lanreotide Autogel $120 \mathrm{mg}$ as primary therapy in acromegaly: results of a prospective multicenter clinical trial. Journal of Clinical Endocrinology and Metabolism 201499 1282-1290. (doi:10.1210/ jc.2013-3318)

8 Neggers SJ, Franck SE, de Rooij FW, Dallenga AH, Poublon RM, Feelders RA, Janssen JA, Buchfelder M, Hofland LJ, Jorgensen JO et al. Long-term efficacy and safety of pegvisomant in combination with long-acting somatostatin analogs in acromegaly. Journal of Clinical Endocrinology and Metabolism 201499 3644-3652. (doi:10.1210/ jc.2014-2032)

9 Trainer PJ, Drake WM, Katznelson L, Freda PU, Herman-Bonert V, van der Lely AJ, Dimaraki EV, Stewart PM, Friend KE, Vance ML et al. Treatment of acromegaly with the growth hormone-receptor antagonist pegvisomant. New England Journal of Medicine 2000342 1171-1177. (doi:10.1056/NEJM200004203421604)

10 Trainer PJ, Ezzat S, D’Souza GA, Layton G \& Strasburger CJ. A randomized, controlled, multicentre trial comparing pegvisomant alone with combination therapy of pegvisomant and long-acting octreotide in patients with acromegaly. Clinical Endocrinology $2009 \mathbf{7 1}$ 549-557. (doi:10.1111/j.1365-2265.2009.03620.x)

11 van der Lely AJ, Bernabeu I, Cap J, Caron P, Colao A, Marek J, Neggers S \& Birman P. Coadministration of lanreotide Autogel and pegvisomant normalizes IGF1 levels and is well tolerated in patients with acromegaly partially controlled by somatostatin analogs alone. European Journal of Endocrinology 2011 164 325-333. (doi:10.1530/EJE10-0867)

12 van der Lely AJ, Hutson RK, Trainer PJ, Besser GM, Barkan AL, Katznelson L, Klibanski A, Herman-Bonert V, Melmed S, Vance ML et al. Long-term treatment of acromegaly with pegvisomant, a growth hormone receptor antagonist. Lancet 2001358 1754-1759. (doi:10.1016/S0140-6736(01)06844-1)

13 Kopchick JJ, List EO, Kelder B, Gosney ES \& Berryman DE. Evaluation of growth hormone $(\mathrm{GH})$ action in mice: discovery of $\mathrm{GH}$ receptor antagonists and clinical indications. Molecular and Cellular Endocrinology 2014386 34-45. (doi:10.1016/j.mce.2013.09.004)

14 Ross RJ, Leung KC, Maamra M, Bennett W, Doyle N, Waters MJ \& Ho KK. Binding and functional studies with the growth hormone receptor antagonist, B2036-PEG (pegvisomant), reveal effects of pegylation and evidence that it binds to a receptor dimer. Journal of Clinical Endocrinology and Metabolism 200186 1716-1723. (doi:10.1210/jc.86.4.1716)

15 Farmacotherapeutisch Kompas. Pegvisomant. Diemen, Amsterdam, Netherlands: Zorginstituut Nederlands, 2016. (Available at: https:// www.farmacotherapeutischkompas.nl/bladeren-volgens-boek/ preparaatteksten/p/pegvisomant) 
16 Neggers SJ, de Herder WW, Janssen JA, Feelders RA \& van der Lely AJ. Combined treatment for acromegaly with long-acting somatostatin analogs and pegvisomant: long-term safety for up to 4.5 years (median 2.2 years) of follow-up in 86 patients. European Journal of Endocrinology 2009160 529-533. (doi:10.1530/EJE-08-0843)

17 Freda PU, Gordon MB, Kelepouris N, Jonsson P, Koltowska-Haggstrom $\mathrm{M} \&$ van der Lely AJ. Long-term treatment with pegvisomant as monotherapy in patients with acromegaly: experience from acrostudy. Endocrine Practices 201521 264-274. (doi:10.4158/EP14330.OR)

18 Neggers SJ, van Aken MO, Janssen JA, Feelders RA, de Herder WW $\&$ van der Lely AJ. Long-term efficacy and safety of combined treatment of somatostatin analogs and pegvisomant in acromegaly. Journal of Clinical Endocrinology and Metabolism 200792 4598-4601. (doi:10.1210/jc.2007-1234)

19 Marazuela M, Lucas T, Alvarez-Escola C, Puig-Domingo M, de la Torre NG, de Miguel-Novoa P, Duran-Hervada A, Manzanares R, Luque-Ramirez M, Halperin I et al. Long-term treatment of acromegalic patients resistant to somatostatin analogues with the GH receptor antagonist pegvisomant: its efficacy in relation to gender and previous radiotherapy. European Journal of Endocrinology 2009160 535-542. (doi:10.1530/EJE-08-0705)

20 Parkinson C, Burman P, Messig M \& Trainer PJ. Gender, body weight, disease activity, and previous radiotherapy influence the response to pegvisomant. Journal of Clinical Endocrinology and Metabolism 200792 190-195. (doi:10.1210/jc.2006-1412)

21 Bernabeu I, Alvarez-Escola C, Quinteiro C, Lucas T, Puig-Domingo M, Luque-Ramirez M, de Miguel-Novoa P, Fernandez-Rodriguez E, Halperin I, Loidi L et al. The exon 3-deleted growth hormone receptor is associated with better response to pegvisomant therapy in acromegaly. Journal of Clinical Endocrinology and Metabolism 201095 222-229. (doi:10.1210/jc.2009-1630)

22 Bianchi A, Mazziotti G, Tilaro L, Cimino V, Veltri F, Gaetani E, Pecorini G, Pontecorvi A, Giustina A \& De Marinis L. Growth hormone receptor polymorphism and the effects of pegvisomant in acromegaly. Pituitary 200912 196-199. (doi:10.1007/s11102-008-0157-8)

23 Filopanti M, Olgiati L, Mantovani G, Corbetta S, Arosio M, Gasco V, De Marinis L, Martini C, Bogazzi F, Cannavo S et al. Growth hormone receptor variants and response to pegvisomant in monotherapy or in combination with somatostatin analogs in acromegalic patients: a multicenter study. Journal of Clinical Endocrinology and Metabolism 201297 E165-E172. (doi:10.1210/jc.2011-1769)

24 Franck SE, van der Lely AJ, Delhanty PJ, Jorgensen JO \& Neggers SJ. Pegvisomant in combination with long-acting somatostatin analogues in acromegaly: the role of the GH receptor deletion of exon 3. European Journal of Endocrinology 2015173 553-561. (doi:10.1530/EJE-15-0519)

25 Franck SE, Broer L, van der Lely AJ, Kamenicky P, Bernabéu I, Malchiodi E, Delhanty PJ, Rivadeneira F \& Neggers SJ. The effect of the growth hormone receptor deletion of exon 3 in acromegaly treated with pegvisomant: a systematic review and meta-analysis. Neuroendocrinology 2016 [Epub ahead of print]. (doi:10.1159/000448844)

26 Petrossians P, Tichomirowa MA, Stevenaert A, Martin D, Daly AF \& Beckers A. The Liege Acromegaly Survey (LAS): a new software tool for the study of acromegaly. Annales $d$ Endocrinologie 201273 190-201. (doi:10.1016/j.ando.2012.05.001)
27 Elmlinger MW, Kuhnel W, Weber MM \& Ranke MB. Reference ranges for two automated chemiluminescent assays for serum insulin-like growth factor I (IGF-I) and IGF-binding protein 3 (IGFBP-3). Clinical Chemistry and Laboratory Medicine 200442 654-664.

28 Madsen M, Fisker S, Feldt-Rasmussen U, Andreassen M, Kristensen LO, Orskov H \& Jorgensen JO. Circulating levels of pegvisomant and endogenous growth hormone during prolonged pegvisomant therapy in patients with acromegaly. Clinical Endocrinology 201480 92-100. (doi:10.1111/cen.12239)

29 Orskov H, Frystyk J, Nielsen C, Hansen AT, Weeke J \& Jorgensen JO. Concomitant, specific determination of growth hormone and pegvisomant in human serum. Growth Hormone and IGF Research 2007 17 431-434. (doi:10.1016/j.ghir.2007.05.001)

30 Sievers C, Baur DM, Schwanke A, Buchfelder M, Droste M, Mann K $\&$ Stalla GK. Prediction of therapy response in acromegalic patients under pegvisomant therapy within the German ACROSTUDY cohort. Pituitary 201518 916-923. (doi:10.1007/s11102-015-0673-2)

31 Domene HM, Marin G, Sztein J, Yu YM, Baron J \& Cassorla FG. Estradiol inhibits growth hormone receptor gene expression in rabbit liver. Molecular and Cellular Endocrinology 1994103 81-87. (doi:10.1016/0303-7207(94)90072-8)

32 Slootweg MC, Swolin D, Netelenbos JC, Isaksson OG \& Ohlsson C. Estrogen enhances growth hormone receptor expression and growth hormone action in rat osteosarcoma cells and human osteoblastlike cells. Journal of Endocrinology 1997155 159-164. (doi:10.1677/ joe.0.1550159)

33 Venken K, Schuit F, Van Lommel L, Tsukamoto K, Kopchick JJ, Coschigano K, Ohlsson C, Moverare S, Boonen S, Bouillon R et al. Growth without growth hormone receptor: estradiol is a major growth hormone-independent regulator of hepatic IGF-I synthesis. Journal of Bone and Mineral Research 200520 2138-2149. (doi:10.1359/ JBMR.050811)

34 Trainer PJ. ACROSTUDY: the first 5 years. European Journal of Endocrinology 2009161 (Supplement 1) S19-S24. (doi:10.1530/EJE-090322)

35 Bianchi A, Valentini F, Iuorio R, Poggi M, Baldelli R, Passeri M, Giampietro A, Tartaglione L, Chiloiro S, Appetecchia M et al. Longterm treatment of somatostatin analog-refractory growth hormonesecreting pituitary tumors with pegvisomant alone or combined with long-acting somatostatin analogs: a retrospective analysis of clinical practice and outcomes. Journal of Experimental and Clinical Cancer Research 201332 40. (doi:10.1186/1756-9966-32-40)

36 Laursen T, Moller J, Fisker S, Jorgensen JO \& Christiansen JS. Effects of a 7-day continuous infusion of octreotide on circulating levels of growth factors and binding proteins in growth hormone (GH)-treated GH-deficient patients. Growth Hormone and IGF Research 19999 451-457. (doi:10.1054/ghir.1999.0131)

37 Pokrajac A, Frystyk J, Flyvbjerg A \& Trainer PJ. Pituitary-independent effect of octreotide on IGF1 generation. European Journal of Endocrinology 2009160 543-548. (doi:10.1530/EJE-08-0822)

38 Jorgensen JO, Feldt-Rasmussen U, Frystyk J, Chen JW, Kristensen LO, Hagen C \& Orskov H. Cotreatment of acromegaly with a somatostatin analog and a growth hormone receptor anatagonist. Journal of Clinical Endocrinology and Metabolism 200590 5627-5631. (doi:10.1210/ jc.2005-0531)

Received 22 November 2016

Revised version received 3 January 2017

Accepted 17 January 2017 